\title{
Diffusivity-Factor Approximation for Spectral Outgoing Longwave Radiation
}

\author{
JiNG FENG AND Yi HUANG \\ Department of Atmospheric and Oceanic Sciences, McGill University, Montreal, Quebec, Canada
}

(Manuscript received 17 August 2018, in final form 13 March 2019)

\begin{abstract}
Accurate integration of directional radiance shows that the conventional diffusivity-factor approximation with a constant diffusivity angle results in an overestimation of the outgoing longwave radiation (OLR) in the window band and an underestimation in the absorption band. We propose an analytical estimation of a spectrally dependent diffusivity angle for clear-sky spectral OLR, considering actual atmospheric conditions and realistic optical path geometry. Beginning with the plane-parallel geometry, we present a new, physical explanation of the conventional diffusivity angle that applies to the gas absorption bands and derives an alternative solution for the window bands. Then a correction scheme is developed to account for the impact of the spherical Earth geometry on the diffusivity angle. The proposed method achieves higher accuracy, reducing biases to generally less than $2 \%$ in all spectral regions.
\end{abstract}

\section{Introduction}

The outgoing longwave radiation (OLR) is a critical variable as it balances the incoming solar (shortwave) radiation and shapes Earth's climate. In essence, OLR is the irradiance flux $F$ contributed by the outgoing radiances $I$ emerging from the top of the atmosphere (TOA) at all possible angles. Their relationship can be expressed as Eq. (1), assuming azimuthal homogeneity:

$$
\begin{aligned}
F(v) & =\int_{0}^{2 \pi} \int_{0}^{\pi / 2} I(v, \theta) \sin (\theta) \cos (\theta) d \theta d \phi \\
& =2 \pi \int_{0}^{\pi / 2} I(v, \theta) \sin (\theta) \cos (\theta) d \theta \\
& =2 \pi \int_{0}^{1} I(v, \mu) \mu d \mu
\end{aligned}
$$

where $\theta$ is the zenith angle, $\phi$ is the azimuth angle, $\mu=\cos (\theta)$, and $v$ denotes the frequency.

It would be computationally expensive to compute OLR by calculating and integrating all the directional radiances. A widely used technique, commonly referred to as the diffusivity-factor approximation (DFA) (Elsasser 1942), is to approximate the OLR as isotropic radiation emerging from an effective zenith angle $\theta_{0}$, so that we can obtain the OLR by simply multiplying the radiance at that angle with a constant $\pi$ :

\footnotetext{
Corresponding author: Jing Feng, jing.feng3@mail.mcgill.ca
}

$$
F(v)=\pi I\left(v, \theta_{0}\right)
$$

Without offering a detailed explanation, Elsasser (1942) first proposed a diffusivity factor $f=1.66$, corresponding to a diffusivity angle of about $53^{\circ}$ according to the relationship $f=1 / \cos \left(\theta_{0}\right)$. As this method became widely used, a few authors explained its mathematical basis. Armstrong (1968) considered this result a feature of broadband OLR resulting from spectral integration, which thus would not apply to the case of spectrally resolved OLR that we are concerned here. Li (2000) showed this method could be considered a one-node Gaussian quadrature approximation (1GQ) of the angular integration. In this paper, we will show that this result can be alternatively derived based on a physical interpretation of the OLR.

With the advancement of measurement technology as well as the improvement of radiation codes, spectrally decomposed radiation has been increasingly used for weather and climate monitoring and model validation (Huang et al. 2007; Feldman et al. 2015; Huang 2013; Bani Shahabadi et al. 2016). When simulating spectrally decomposed OLR fluxes from an atmospheric profile, an adequate radiance-to-irradiance conversion scheme is required. The DFA method with a constant diffusivity angle is often used in such applications to reduce computing costs (e.g., Huang et al. 2013; Pan and Huang 2018). However, the DFA method, although widely used for the spectrally integrated OLR, is not validated 
for the spectrally decomposed irradiance fluxes. As shown in the following sections, using constant-angle DFA across the longwave spectrum (100-2800 $\mathrm{cm}^{-1}$ ) would lead to considerable biases. Other authors have discussed relevant problems. For instance, Mehta and Susskind (1999) used empirically determined diffusivity angles for different spectral bands that are independent of atmospheric condition. Zhao and Shi (2013) proposed a bridge function-based scheme to calculate the diffusivity angle according to the optical depth. In general, these methods were based on the consideration of angular integration of radiance in an isolated atmospheric layer and thus do not account the geometry of the line of sight, which in reality is complicated, for instance, by the curvature of Earth's surface.

In this paper, based on our physical interpretation of the DFA, we propose a method for determining the diffusivity angle at every frequency according to specific atmospheric conditions. This method treats the window band (weak atmospheric absorption) and gas absorption bands (strong atmospheric absorption) differently, which as shown below is necessary according to the physical interpretation of the DFA method. We also provide a scheme that corrects the bias due to the path geometry. The paper is structured as follows: in section 2, we present our physical interpretation of the DFA method; in section 3, we adjust the scheme to account for the effect of spherical Earth surface geometry; the performance and implication of this method are demonstrated in section 4 .

\section{Physical model}

The monochromatic radiance from viewing angle $\theta$ at spectral frequency of $v$ is (Goody and Yung 1989)

$$
\begin{aligned}
I(v, \theta) & =I_{\mathrm{atm}}+I_{\text {srf }} \\
& =\int_{0}^{\tau_{s}} B[v, T(\tau)] e^{-\tau / \mu} d\left(\frac{\tau}{\mu}\right)+B\left[v, T\left(\tau_{s}\right)\right] e^{-\tau_{s} / \mu},
\end{aligned}
$$

where $\tau$ is the nadir optical depth from TOA to a vertical level, and $\tau_{s}$ is the total optical depth from TOA to the surface. The term $B[v, T(\tau)]$ is the Plank function of blackbody radiation at wavenumber $v$. The first term of Eq. (3) corresponds to the atmospheric contribution $I_{\text {atm }}$, and the second term corresponds to the surface contribution $I_{\text {srf }}$. Note this equation holds in a nonscattering plane-parallel atmosphere when both path refraction and Earth surface curvature are neglected.

To proceed, let us first relate the source function $B(v, T)$ to the optical depth $\tau$. According to Planck function, the blackbody emission $B(v, T)$ is

$$
\begin{aligned}
B(v, T) & =\frac{2 h v^{3}}{c^{2}} \frac{1}{e^{h v /\left(k_{B} T\right)}-1} \\
& =B\left(v, T_{0}\right)+\left.\frac{\partial B(v, T)}{\partial T}\right|_{T_{0}}\left(T-T_{0}\right)+O\left(T-T_{0}\right)^{2} \\
& =B\left(v, T_{0}\right)\left[1-\beta\left(T_{0}-T\right)\right]+O\left(T_{0}-T\right)^{2}
\end{aligned}
$$

where $\left.\beta \equiv\left[1 / B\left(v, T_{0}\right)\right](\partial B / \partial T)\right|_{T_{0}}$. This approximation holds well throughout the infrared spectra, as shown by Fig. 1a, although the second-order residual becomes larger at higher wavenumber.

In a standard atmospheric condition, we can assume a constant temperature lapse rate $\Gamma$ that satisfies $T(z)=$ $T_{0}-\Gamma z$. Note that the vertical distribution of optical depth follows a similar exponentially decaying pattern to gas absorber concentration:

$$
\begin{aligned}
\tau(z, v) & =\tau_{s}(v) e^{-z / H_{\tau(v)}} \\
z(\tau) & =-H_{\tau} \ln \left(\frac{\tau}{\tau_{s}}\right) .
\end{aligned}
$$

The term $H_{\tau}$ is the scale height of optical depth that is spectrally dependent on the vertical distribution of the absorber. The temperature is now linearly dependent on $\ln (\tau)$ :

$$
T(\tau)=T_{0}+\Gamma H_{\tau} \ln \left(\frac{\tau}{\tau_{0}}\right) .
$$

We can then relate the blackbody emission of each layer to the optical depth $\tau$, given Eq. (4):

$$
B[v, T(\tau)]=B\left(v, T_{0}\right)\left(1+\alpha \ln \frac{\tau}{\tau_{0}}\right),
$$

where the parameter $\alpha=\beta \Gamma H_{\tau}$. The term $\tau_{0}$ is the reference optical depth at which level the reference temperature $T_{0}$ locates. This relationship [Eq. (7)] is examined in Fig. $1 \mathrm{~b}$ at $700 \mathrm{~cm}^{-1}$ (in a $\mathrm{CO}_{2}$ absorption band). In this comparison, the temperature profile $T(\tau)$ at each optical layer is generated from Eq. (6), using the reference optical depth $\tau_{0}=1$ for validation. Figure $1 \mathrm{~b}$ shows that Eq. (7) approximates the source function from surface to TOA very well.

Next, following Eq. (3), we numerically evaluate the nadir-view radiance $I(v, \theta=0)$ as a weighted sum of source functions from all discretized layers (Goody and Yung 1989):

$$
I(v, \theta=0)=\sum_{i} B\left[v, T\left(\tau_{i}\right)\right] W\left(\tau_{i}\right) .
$$



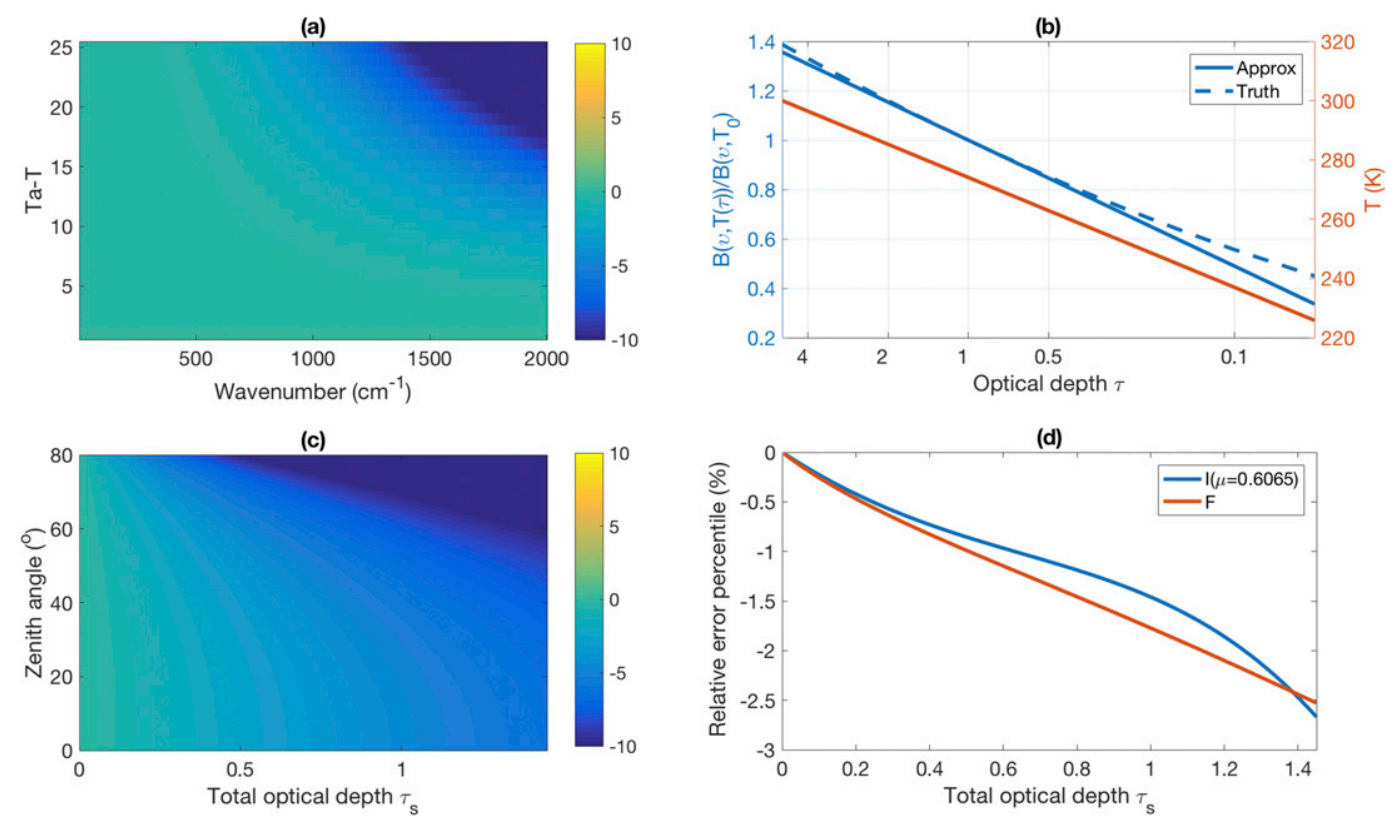

FIG. 1. Validation of the approximations of (a) source function $B$ as a linear function of temperature, (b) source function-optical depth $(B-\tau)$ relationship in the absorption band, (c) atmospheric emission $I_{\mathrm{atm}}$, and (d) directional radiance $I$ at viewing angle $\theta=\arccos \left(e^{-1 / 2}\right)$ and irradiance flux $F$ in the window band. Shown in (a) are the errors $(\%)$ in the linearization of the Planck function, defined as the fractional difference between the approximation of the source function according to Eq. (4) and the truth computed from the Planck function. Shown in (b) are the source function accurately computed from the atmospheric profile (truth) and that approximated according to Eq. (7); the temperature profile used in this validation is plotted as a red line, showing a linear dependence on $\ln (\tau)$, as Eq. (6) indicates. Shown in (c) are the errors (\%) in atmospheric emission, defined as the fractional difference between the approximation based on Eq. (18) and the accurately integrated source function according to Eq. (3). Shown in (d) are the fractional errors of directional radiance $I$ at $\mu=e^{-1 / 2}$ and irradiance flux $F$ approximated according to Eq. (21) for $I$ and Eq. (22) for $F$, respectively; the truth is computed from Eq. (3) for $I$ and Eq. (1) for $F$.

The term $W$ is the so-called weighting function, which is the derivative of transmission function with respect to altitude $z$ :

$$
W \equiv \frac{\partial e^{-\tau}}{\partial z}
$$

The weighting functions in the gas absorption bands are typically bell shaped and peak at a level where $\tau=1$ (Goody and Yung 1989; Huang and Bani Shahabadi 2014). The outgoing radiance largely originates from this level. Therefore, we consider the reference atmospheric temperature $T_{0}$ in Eq. (7) to be the temperature at this emission layer, so that Eq. (7) becomes

$$
B[v, T(\tau)]=B\left(v, T_{0}\right)[1+\alpha \ln (\tau)] .
$$

Following the emission-layer displacement model (ELDM) (Huang and Bani Shahabadi 2014), let us consider a set of weighting function in the nadir case: $W_{1}, W_{2}, \ldots, W_{n}$, which correspond to optical depths $\tau_{1}, \tau_{2}, \ldots, \tau_{n}$. Because $W$ is only a function of $\tau$, in the limb-view case, the same set of $W_{i}$ values are taken at $\mu \tau_{1}, \mu \tau_{2}, \ldots, \mu \tau_{n}$; that is, the layer with the same weighting function value is displaced to a higher altitude. We rewrite the Eq. (8) as

$$
I(v, \mu)=\sum_{i} B\left[v, T\left(\mu \tau_{i}\right)\right] W_{i}
$$

so that

$$
I(v, \mu)=B\left(v, T_{0}\right) \sum_{i}\left[1+\alpha \ln \left(\tau_{i}\right)+\alpha \ln \mu\right] W_{i} .
$$

Based on Eq. (1), the OLR at this frequency can be obtained by integration of Eq. (12) over $\mu$ :

$$
\begin{aligned}
F(v) & =2 \pi \int_{0}^{1} I(v, \mu) \mu d \mu \\
& =\pi B\left(v, T_{0}\right) \sum_{i}\left[1+\alpha \ln \left(\tau_{i}\right)-\frac{\alpha}{2}\right] W_{i} .
\end{aligned}
$$

Combining Eqs. (2), (12), and (13), 


$$
\begin{aligned}
F(v) & =\pi I\left(v, \mu_{0}\right) \\
\sum_{i}\left[1+\alpha \ln \left(\tau_{i}\right)-\frac{\alpha}{2}\right] W_{i} & =\sum_{i}\left[1+\alpha \ln \left(\tau_{i}\right)+\alpha \ln \left(\mu_{0}\right)\right] W_{i} .
\end{aligned}
$$

We find

$$
\mu_{0}=e^{-1 / 2} \approx 0.6065
$$

This solution $\mu_{0}=e^{-1 / 2}$ is the same as what Armstrong (1968) and $\mathrm{Li}$ (2000) obtained, although it is based on a different physical interpretation of the OLR. This solution largely results from the temperature to optical depth relation in Eq. (7), which only needs to hold over a limited vertical range that the weighting function primarily spans.

The physical derivation above also indicates under what condition the DFA as given by Eq. (15) would fail: when the ELDM does not apply. Most notably, Eq. (12) assumes the surface contribution $I_{\text {srf }}$ to be negligible. This is not applicable to the window band, where the surface contribution dominates the TOA flux. Interestingly, the previous authors noted a breakdown of the DFA under similar circumstances, that is, when the optical depth $\tau$ approaches zero (Li 2000; Zhao and Shi 2013). In the following section, we will use a different approach to find an appropriate diffusivity angle in the window band.

\section{Window band model}

Because of its sensitivity to the surface climate, the radiance in the window is especially important for climate monitoring. As addressed in the previous section, the ELDM poorly represents the window band. In this section, we focus on finding an approach better suited for the weak atmospheric absorption feature in the window band.

In the window band, the surface emission is a primary contributor to the TOA flux. With an elevated viewing angle, which creates a longer optical path, the atmosphere becomes more opaque, leading to more contribution from the atmosphere and less from the surface. Thus both atmospheric and surface effects are nonnegligible.

Adopting a "one layer" atmosphere model, we rewrite Eq. (3) as

$$
\begin{aligned}
I(v, \mu) & =I_{\mathrm{atm}}+I_{\mathrm{srf}} \\
& =B\left[v, T_{a}(\mu)\right]\left(1-e^{-\tau_{s}^{\prime} / \mu}\right)+B\left(v, T_{S}\right) e^{-\tau_{s}^{\prime} / \mu},
\end{aligned}
$$

where the term $B\left[v, T_{a}(\mu)\right]\left(1-e^{-\tau_{s} / \mu}\right)$ represents the emission of the whole atmospheric column, which is equivalent to the first term in Eq. (3).
Because the optical depth $\tau_{s}$ in the window band is typically much less than unity, the atmospheric effective emission temperature $T_{a}$ is close to surface temperature $T_{s}$. Hence, we can set the reference temperature $T_{0}$ in Eq. (7) to be $T_{s}$ and the reference optical depth $\tau_{0}=\tau_{s}$ :

$$
\begin{aligned}
B[v, T(\tau)] & =B\left(v, T_{s}\right)\left[1+\alpha \ln \left(\frac{\tau}{\tau_{s}}\right)\right] \\
B\left[v, T_{a}(\mu)\right] & =\int_{0}^{\tau_{s}} B[v, T(\tau)] \frac{e^{-\tau / \mu}}{1-e^{-\tau_{s} / \mu}} d \frac{\tau}{\mu} \\
& =B\left(v, T_{s}\right) \int_{0}^{\tau_{s}}\left(1+\alpha \ln \frac{\tau}{\tau_{s}}\right) \frac{e^{-\tau / \mu}}{1-e^{-\tau_{s} / \mu}} d \frac{\tau}{\mu} .
\end{aligned}
$$

This integration cannot be analytically solved. However, it can be approximated as a linear function on $\tau_{s} / \mu$ if we linearized the term $e^{-\tau / \mu} /\left(1-e^{-\tau_{s} / \mu}\right)$, considering both $\tau_{s} / \mu$ and $\tau / \mu$ are small:

$$
\begin{aligned}
B\left[v, T_{a}(\mu)\right] & \approx B\left(v, T_{s}\right) \int_{0}^{\tau_{s}}\left(1+\alpha \ln \frac{\tau}{\tau_{s}}\right) \frac{e^{-\tau / \mu}}{\frac{\tau_{s}}{\mu}-\frac{\tau_{s}^{2}}{2 \mu^{2}}} d \frac{\tau}{\mu} \\
& \approx B\left(v, T_{s}\right) \int_{0}^{\tau_{s}}\left(1+\alpha \ln \frac{\tau}{\tau_{s}}\right) e^{-\tau / \mu}\left(\frac{\mu}{\tau_{s}}+\frac{1}{2}\right) d \frac{\tau}{\mu} \\
& \approx B\left(v, T_{s}\right) \int_{0}^{\tau_{s}}\left(1+\alpha \ln \frac{\tau}{\tau_{s}}\right)\left(\frac{\mu}{\tau_{s}}+\frac{1}{2}-\frac{\tau}{\tau_{s}}\right) d \frac{\tau}{\mu} \\
& =B\left(v, T_{s}\right)\left(1-\frac{\alpha}{4} \frac{\tau_{s}}{\mu}-\alpha\right)
\end{aligned}
$$

It means that, considering the limb-view path and inhomogeneity in the atmospheric column, the effective atmospheric emission is affected by $\alpha$ and $\tau_{s} / \mu$. To demonstrate the validity of this result, we set up a simulation test, using the atmospheric condition described in Eqs. (5) and (6), to compute the limb-view radiance from $0^{\circ}$ to $89^{\circ}$ and angularly integrate them to obtain the irradiance at $100 \mathrm{~km}$ above the ground. Prescribed in the computation are a surface temperature of $300 \mathrm{~K}$ and atmospheric temperatures continuously decreasing from this value at a lapse rate $\Gamma$ of $6.5 \mathrm{~K} \mathrm{~km}^{-1}$. As shown in Fig. 1c, this approximation [Eq. (18)] generally yields error within $5 \%$ in the window band compared to the truth $\left[I_{\mathrm{atm}}\right.$ computed according to Eq. (3)]. The residual becomes larger with increasing viewing angle $\theta$ and the total optical depth $\tau_{s}$ because of the small $\tau_{s} / \mu$ assumption in Eq. (18).

If we substitute Eq. (18) back into Eq. (16), the window band directional radiance and flux become 


$$
\begin{aligned}
I(v, \mu)= & B\left(v, T_{a}, \mu\right)\left(1-e^{-\tau_{s} / \mu}\right)+B\left(v, T_{s}\right) e^{-\tau_{s} / \mu} \\
= & B\left(v, T_{s}\right)\left[-\frac{1}{4 \mu} \alpha \tau_{s}+(1-\alpha)\right. \\
& \left.+\frac{1}{4 \mu} \alpha \tau_{s} e^{-\tau_{s} / \mu}+\alpha e^{-\tau_{s} / \mu}\right], \\
F(v)= & 2 \pi \int_{0}^{1} I(v, \mu) \mu d \mu \\
= & \pi B\left(v, T_{s}\right)\left[1-\alpha-\frac{1}{2} \alpha \tau_{s}+\frac{\alpha}{2} \tau_{s}^{2} E_{1}\left(\tau_{s}\right)\right. \\
& \left.+\alpha e^{-\tau_{s}}-\frac{1}{2} \alpha \tau_{s} e^{-\tau_{s}}\right],
\end{aligned}
$$

where $E_{1}=\int_{0}^{1} \mu e^{-\tau_{s} / \mu} d \mu$. For the simplicity of the solution, we apply a Taylor series expansion at $\tau_{s}=0$ to both $I(v, \mu)$ and $F(v)$ and keep the third-order term based on validation against $\tau_{s}$ value in the range of $[0,1.5]$; meanwhile, we eliminate the third-order term of $\mu$ in a Taylor series expansion at $\mu=0.5$.

$$
\begin{aligned}
\frac{I(v, \mu)}{B\left(v, T_{s}\right)} \approx 1-\frac{\alpha \tau_{s}}{\mu}+\frac{\alpha \tau_{s}^{2}}{4 \mu^{2}}+\frac{\alpha \tau_{s}^{3}}{24 \mu^{3}}+O\left(\tau_{s}^{4}\right) \\
\approx 1-\frac{1}{3} \alpha \tau_{s}^{3}+\alpha \frac{\tau_{s}^{3}-2 \tau_{s}}{2 \mu}+\alpha \frac{\tau_{s}^{2}-\tau_{s}^{3}}{4 \mu^{2}}, \\
\frac{F(v)}{\pi B\left(v, T_{s}\right)} \approx 1-2 \alpha \tau_{s}-\frac{1}{2} \alpha \tau_{s}^{2}\left[\gamma+\ln \left(\tau_{s}\right)-2\right] \\
+\frac{1}{12} \alpha \tau_{s}^{3}+O\left(\tau_{s}^{4}\right)
\end{aligned}
$$

where $\gamma \approx 0.5772$ is the Euler's constant. We validate the two approximations in Fig. 1d. It shows that, in the window band, both approximations yield errors less than $4 \%$, based on which an accurate diffusivity angle can be inferred.

Combining Eqs. (2) and (18), we obtain an equation for the diffusivity angle $\mu_{0}$ :

$$
\begin{aligned}
& \frac{I\left(v, \mu_{0}\right)}{B\left(v, T_{s}\right)}=\frac{F(v)}{\pi B\left(v, T_{s}\right)}, \\
& 1-\frac{1}{3} \alpha \tau_{s}^{3}+\alpha \frac{\tau_{s}^{3}-2 \tau_{s}}{2 \mu}+\alpha \frac{\tau_{s}^{2}-\tau_{s}^{3}}{4 \mu^{2}} \\
& \quad=1-2 \alpha \tau_{s}-\frac{1}{2} \alpha \tau_{s}^{2}\left[\gamma+\ln \left(\tau_{s}\right)-2\right]+\frac{1}{12} \alpha \tau_{s}^{3},
\end{aligned}
$$

The problem then becomes solving a quadratic equation of $\mu_{0}$ :

$$
\begin{aligned}
a \mu_{0}^{2}+b \mu_{0}+c=0 & \\
a & =-2 \alpha \tau_{s}-\frac{1}{2} \alpha \tau_{s}^{3}\left(\gamma+\ln \tau_{s}-2\right)+\frac{5}{12} \alpha \tau_{s}^{2}, \\
b & =\alpha \tau_{s}-\frac{1}{2} \alpha \tau_{s}^{3}, \\
c & =-\frac{\alpha \tau_{s}^{2}}{4}+\frac{\alpha \tau_{s}^{3}}{4}, \\
\cos \left(\theta_{0}\right) & =\mu_{0}=\frac{-b-\sqrt{b^{2}-4 a c}}{2 a}, \quad \tau_{s} \leq 1.4705 .
\end{aligned}
$$

The limit, $\tau_{s} \leq 1.4705$, is chosen so that the solution is continuous with our absorption band solution, $\mu_{0}=e^{-1 / 2}$ when $\tau_{s}=1.4705$. The solution can be further simplified to

$$
\mu_{0} \approx \frac{1}{2}-\frac{\tau_{s}}{8}\left(\gamma+\ln \tau_{s}\right)
$$

when optical depth $\tau_{s} \leq e^{-1-\gamma}=0.2066$.

To validate the above result [Eq. (24)], we set a simple radiative transfer model that accurately computes the source function of each atmospheric layer and vertically integrates it according to Eq. (3). The directional radiance is obtained at each $0.01^{\circ}$ between $0^{\circ}$ and $89.99^{\circ}$, from which the irradiance flux is integrated. We then match the directional radiance and irradiance to find the $\theta_{0}$ exhibiting the smallest error according to Eq. (2). As shown in Fig. 2a, we affirm that the spectrally dependent DFA obtained in Eq. (24) has a noticeable improvement compared to the constant-angle DFA method, especially in the extremely transparent spectral regime. In contrast to the constant-angle DFA in absorption bands, it is now dependent on the total optical depth $\tau_{s}$, although the atmospheric properties, such as the lapse rate $\Gamma$, are still not relevant.

\section{Spherical correction}

In the previous discussion, a plane-parallel geometry is assumed, so that the zenith angle $\theta$ from the surface level to the TOA is constant along the line of sight. As shown in Fig. 3, in reality, the effective zenith angle $\theta^{\prime}$ varies with altitude $z$ and becomes larger farther away from the observer, resulting in an extended geometry path compared to plane-parallel geometry, because of the curvature of Earth's surface.

Considering the spherical geometry, the effective zenith angle $\theta^{\prime}$ can be expressed as a function of height $z$ : 
(a)

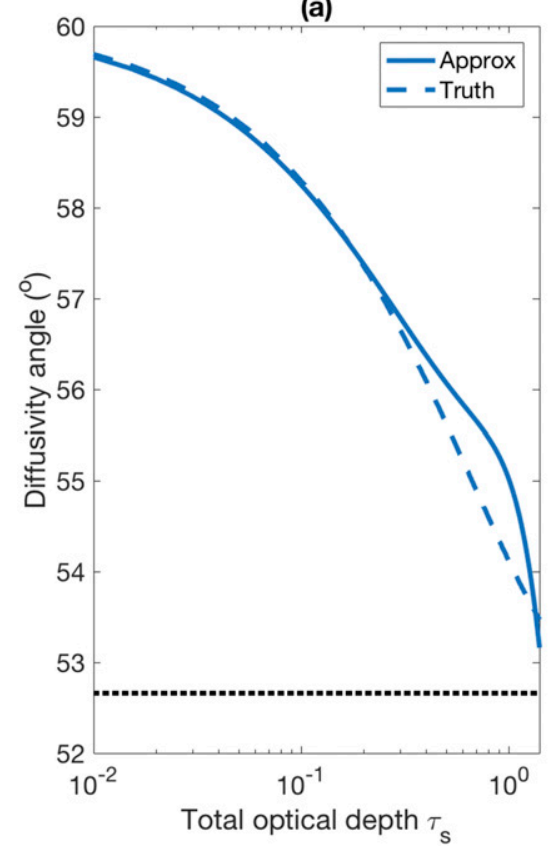

(b)

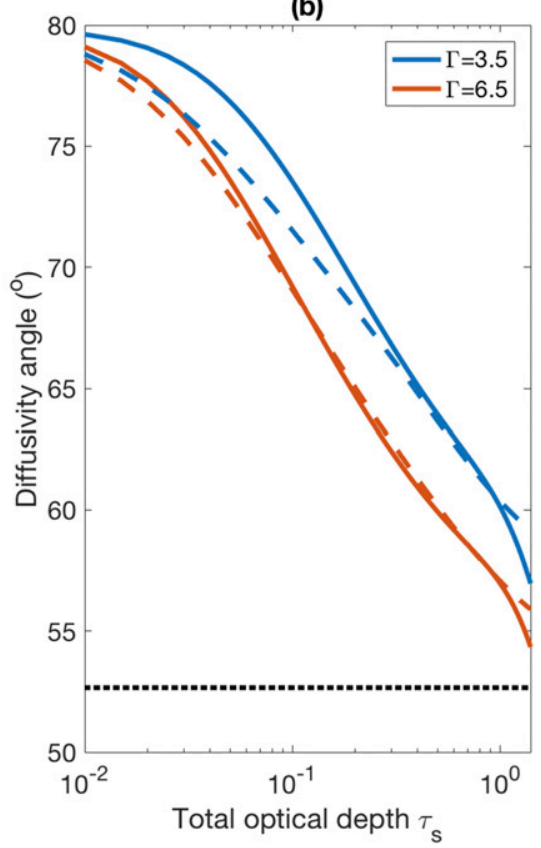

FIG. 2. Diffusivity angle considering (a) plane-parallel and (b) realistic path geometry. In (a), the approximation [Eq. (24)] and truth are shown as solid and dashed lines, respectively. In (b), approximation [Eq. (29)] and truth are shown as solid and dashed lines, while different atmospheric lapse rates $\left(\mathrm{K} \mathrm{km}^{-1}\right)$ are indicated by color. The dotted line is the constant diffusivity angle at $52.66^{\circ}$.

$$
\mu^{\prime}(z)^{2}=\cos \left[\theta^{\prime}(z)\right]^{2}=1-\left(1-\mu^{2}\right)\left(\frac{R+H}{R+z}\right)^{2} .
$$

Because the TOA irradiance flux is mostly contributed by emission layers at height $z_{0}$, we can approximate the zenith angle $\theta^{\prime}$ with its value at this layer, so that

$$
\mu^{\prime 2}=\cos \left(\theta^{\prime}\right)^{2} \approx 1-\left(1-\mu^{2}\right)\left(\frac{R+H}{R+z_{0}}\right)^{2}
$$

The emission level, as discussed in the previous section, is at $\tau=1$ in the absorption band and at the surface in the window band. According to the $\tau-z$ relationship described in Eq. (5), we have $z_{0}\left(\tau=\tau_{s}\right)=$ 0 in the window band and $z_{0}(\tau=1)=H_{\tau} \ln \left(\tau_{s}\right)$ in the absorption band. Note that the latter one can be directly referred from the optical depth profile with a forward model.

Substituting Eq. (27) into Eq. (1), we find the spectral flux in Eqs. (22) and (13) can be adjusted with a factor $\left[\left(R+z_{0}\right) /(R+H)\right]^{2}$ to account for the effect of Earth geometry, as shown below:

$$
\begin{aligned}
F_{c}(v) & =2 \pi \int_{0}^{1} I\left(v, \mu^{\prime}\right) \mu d \mu \\
& =2 \pi \int_{0}^{1} I\left(v, \mu^{\prime}\right) \mu^{\prime} d \mu^{\prime} \frac{\mu d \mu}{\mu^{\prime} d \mu^{\prime}} \\
& \approx\left(\frac{R+z_{0}}{R+H}\right)^{2} F(v) .
\end{aligned}
$$

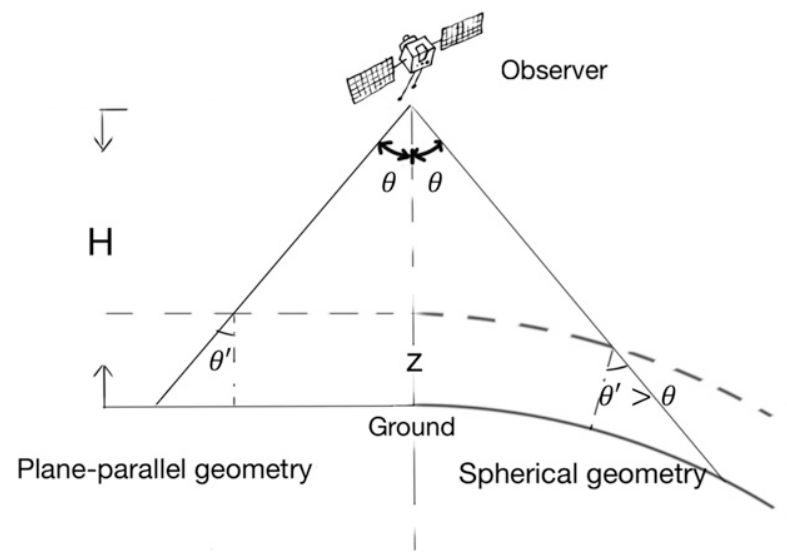

FIG. 3. Path zenith angle affected by Earth surface curvature. $H$ is the observer height, and $z$ is the emission-layer height. $\theta$ and $\theta^{\prime}$ are the zenith angles at observer height $H$ and the emission-layer $z$, respectively. 
TABLE 1. Errors $\left(\mathrm{W} \mathrm{m}^{-2}\right)$ in five spectral bands $\left(\mathrm{cm}^{-1}\right)$ caused by a constant diffusivity angle $\left(\mu_{0}=e^{-1 / 2}\right)$, double Gaussian quadrature (2GQ), and the spectrally dependent diffusivity angle $\mu_{0}(v)$, considering plane-parallel (p-p) and realistic path (realistic) geometry, respectively.

\begin{tabular}{|c|c|c|c|c|c|c|c|c|c|c|c|}
\hline \multirow[b]{2}{*}{ Atmosphere type } & \multirow[b]{2}{*}{ Method } & \multicolumn{2}{|c|}{$[581,760]$} & \multicolumn{2}{|c|}{$[760,1000]$} & \multicolumn{2}{|c|}{$[1001,1060]$} & \multicolumn{2}{|c|}{$[1061,1230]$} & \multicolumn{2}{|c|}{$[1305,1800]$} \\
\hline & & $\mathrm{p}-\mathrm{p}$ & Realistic & $\mathrm{p}-\mathrm{p}$ & Realistic & $\mathrm{p}-\mathrm{p}$ & Realistic & $\mathrm{p}-\mathrm{p}$ & Realistic & p-p & Realistic \\
\hline \multirow[t]{3}{*}{ Standard } & $\mu_{0}(v)$ & -0.47 & -0.47 & -0.06 & 0.04 & -0.23 & -0.26 & -0.09 & 0.19 & -0.13 & -0.01 \\
\hline & $\mu_{0}=e^{-1 / 2}$ & -0.47 & -0.50 & 0.38 & 1.77 & -0.23 & -0.24 & 0.21 & 0.74 & -0.13 & -0.08 \\
\hline & $2 \mathrm{GQ}$ & 0.03 & -0.05 & 0.01 & 0.88 & 0.04 & -0.03 & 0.01 & 0.33 & 0.01 & 0.02 \\
\hline \multirow[t]{3}{*}{ Tropical } & $\mu_{0}(v)$ & -0.49 & -0.37 & -0.10 & 0.37 & -0.20 & -0.23 & -0.08 & 0.30 & -0.13 & 0.00 \\
\hline & $\mu_{0}=e^{-1 / 2}$ & -0.49 & -0.48 & 0.27 & 1.95 & -0.20 & -0.19 & 0.21 & 0.88 & -0.13 & 0.08 \\
\hline & $2 \mathrm{GQ}$ & 0.03 & -0.03 & 0.04 & 1.01 & 0.05 & -0.03 & 0.01 & 0.40 & 0.01 & 0.06 \\
\hline \multirow[t]{3}{*}{ Midlatitude summer } & $\mu_{0}(v)$ & -0.49 & -0.44 & -0.03 & 0.32 & -0.26 & -0.29 & -0.06 & 0.26 & -0.14 & 0.02 \\
\hline & $\mu_{0}=e^{-1 / 2}$ & -0.49 & -0.52 & 0.36 & 1.83 & -0.26 & -0.28 & 0.21 & 0.79 & -0.14 & -0.06 \\
\hline & 2GQ & 0.03 & -0.05 & 0.01 & 0.90 & 0.05 & -0.03 & 0.01 & 0.36 & 0.01 & 0.03 \\
\hline \multirow[t]{3}{*}{ Midlatitude winter } & $\mu_{0}(v)$ & -0.37 & -0.36 & -0.02 & 0.24 & -0.18 & -0.19 & -0.05 & 0.17 & -0.08 & 0.01 \\
\hline & $\mu_{0}=e^{-1 / 2}$ & -0.37 & -0.37 & 0.21 & 1.26 & -0.18 & -0.19 & 0.14 & 0.52 & -0.08 & -0.04 \\
\hline & $2 \mathrm{GQ}$ & 0.03 & -0.04 & 0.01 & 0.68 & 0.03 & -0.02 & 0.00 & 0.25 & 0.01 & 0.03 \\
\hline \multirow[t]{3}{*}{ High-latitude summer } & $\mu_{0}(v)$ & -0.46 & -0.49 & -0.07 & -0.01 & -0.25 & -0.29 & -0.08 & 0.15 & -0.12 & -0.02 \\
\hline & $\mu_{0}=e^{-1 / 2}$ & -0.46 & -0.52 & 0.35 & 1.54 & -0.25 & -0.28 & 0.18 & 0.65 & -0.12 & -0.08 \\
\hline & $2 \mathrm{GQ}$ & 0.03 & -0.06 & 0.00 & 0.73 & 0.04 & -0.03 & 0.01 & 0.29 & 0.01 & 0.01 \\
\hline \multirow[t]{3}{*}{ High-latitude winter } & $\mu_{0}(v)$ & -0.27 & -0.29 & 0.00 & 0.33 & -0.11 & -0.12 & -0.02 & 0.15 & -0.05 & 0.03 \\
\hline & $\mu_{0}=e^{-1 / 2}$ & -0.27 & -0.26 & 0.11 & 0.83 & -0.11 & -0.12 & 0.08 & 0.33 & -0.05 & -0.00 \\
\hline & $2 \mathrm{GQ}$ & 0.02 & -0.03 & 0.01 & 0.47 & 0.02 & -0.01 & 0.00 & 0.17 & 0.01 & 0.02 \\
\hline
\end{tabular}

It means that the surface geometry generally decreases the irradiance flux at TOA, resulted from an elevated emission layer, or a decreased contribution from the surface in the window band. This does not apply to the strong absorption band as the emission layer approaches the stratosphere, where the surface geometry leads to a slightly negative or even positive difference.

Now the solution for window band in Eq. (24) becomes

$$
\begin{gathered}
\mu_{0}^{\prime}=\frac{-b-\sqrt{b^{2}-4 a c}}{2 a}, \quad \tau_{s}<1.4705, \\
\cos \left(\theta_{0}\right)=\mu_{0} \approx \sqrt{1-\left(1-\mu_{0}^{\prime 2}\right)\left(1-\frac{H}{R+H}\right)^{2}},
\end{gathered}
$$

where

$$
\left\{\begin{array}{l}
a=\left\{1-2 \alpha \tau_{s}-\frac{\alpha \tau_{s}^{2}}{2}\left[r+\ln \left(\tau_{s}\right)-2\right]+\frac{\alpha \tau_{s}^{3}}{12}\right\}\left(1-\frac{H}{R+H}\right)^{2}-1+\frac{\alpha \tau_{s}^{3}}{3} \\
b=\alpha \tau_{s}-\frac{1}{2} \tau_{s}^{3} \\
c=-\frac{\alpha \tau_{s}^{2}}{4}+\frac{\alpha \tau_{s}^{3}}{4}
\end{array}\right.
$$

An adjustment for absorption band is offered here as well:

$$
\begin{aligned}
\mu_{0}^{\prime} & =e^{-1 / 2} \\
\cos \left(\theta_{0}\right) & =\mu_{0} \approx \sqrt{1-\left(1-\mu_{0}^{\prime 2}\right)\left[1-\frac{H-z_{0}(\tau=1)}{R+H}\right]^{2}} .
\end{aligned}
$$

In contrast to previous results, where the atmospheric condition is not relevant in the solution, now $\alpha$ becomes an important factor influencing the outgoing irradiance when the total optical depth is small. This result illustrates that, aside from optical properties, the choice of diffusivity angle is also dependent on atmospheric conditions, such as surface temperature and lapse rate, which determine $\alpha$. This is a factor ignored in previous studies.

Following a similar simulation test described in previous section, Fig. 2 b reveals that, with the effect of the surface curvature, the choice of diffusivity angle decreases with an increasing $\Gamma$, accounting for the effect of increasing $\alpha$; similar dependence can be found on the wavenumber $v$ and $1 / T_{s}$ as well. In contrast to 
(a)

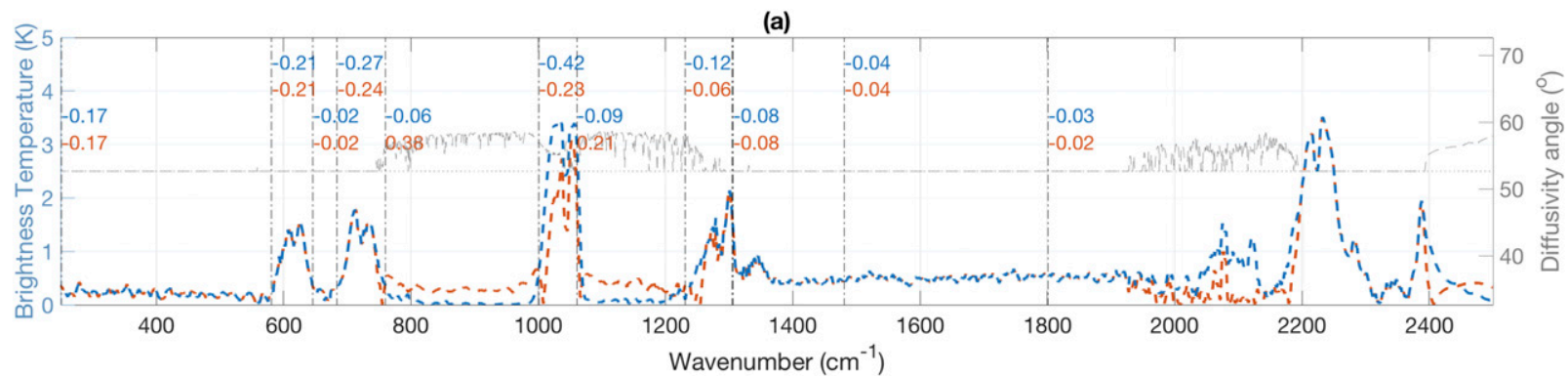

(b)

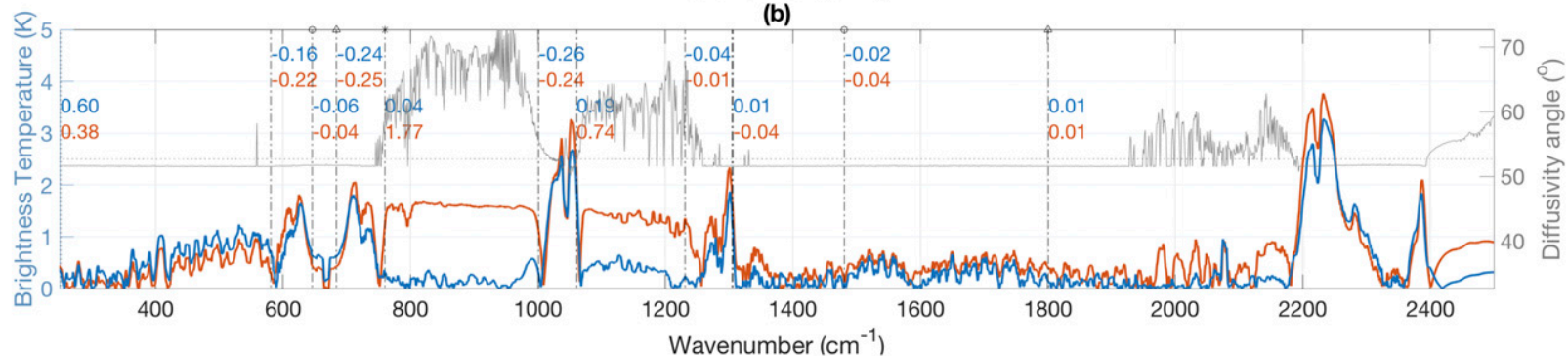

(c)

(d)

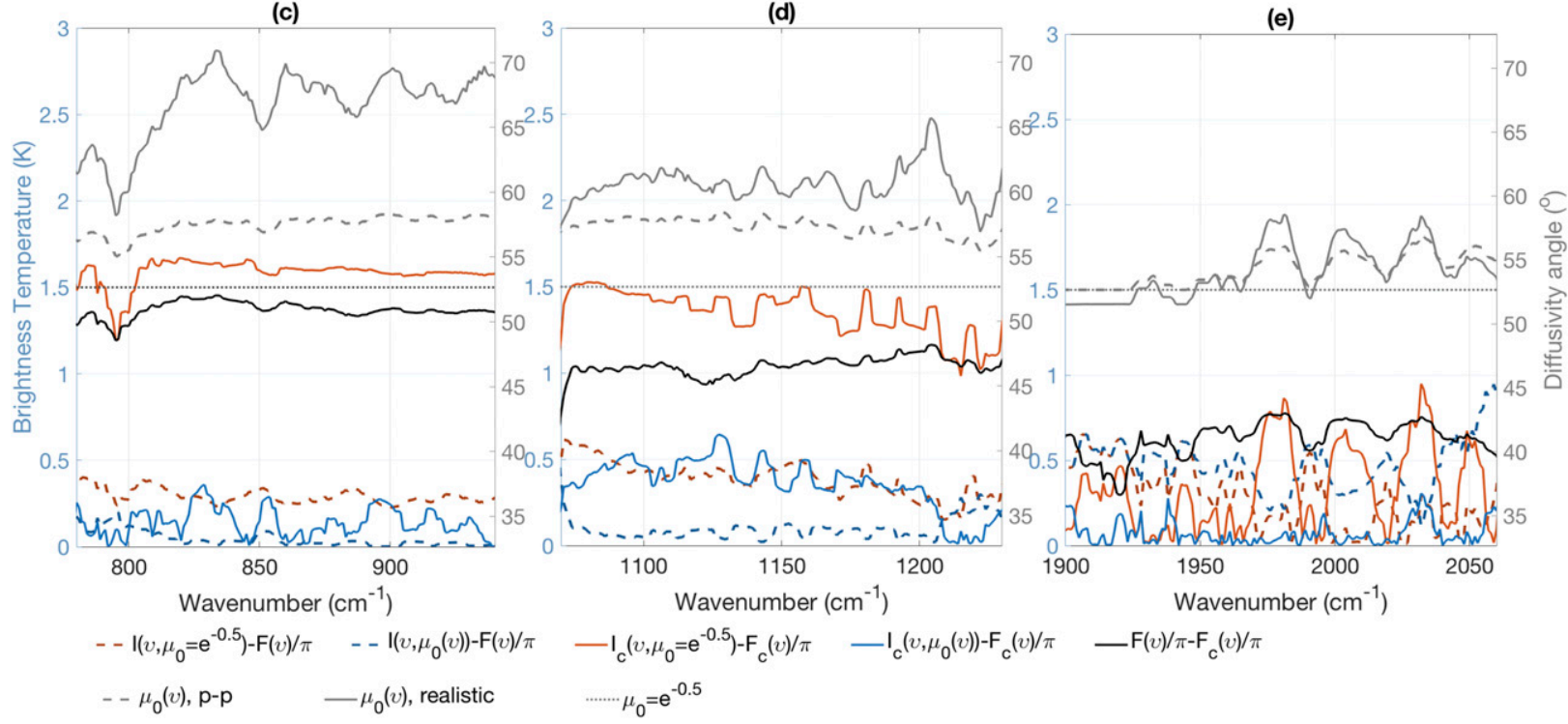

FIG. 4. Absolute error of irradiance flux [expressed by blackbody brightness temperature (K)] induced by constant diffusivity angle (red, $\mu_{0}=e^{-0.5}$ ) and spectrally dependent diffusivity angle [blue, $\mu_{0}(v)$ ] in (a) plane-parallel geometry [dashed; Eq. (24)] and (b) realistic path [solid; Eq. (29)] in the full infrared spectral range and (c)-(e) in three optically thin bands. Shown by the solid black curve is the flux difference [between $F(v)$ in plane-parallel and $F_{c}(v)$ in realistic geometry] caused by Earth's surface geometry. The gray line is the diffusivity angle, corresponding to the $y$ axis on the right. These results are smoothed using a median filter of width $=10 \mathrm{~cm}^{-1}$.

the conventional approach, the diffusivity angle in an extremely transparent band can be as high as $80^{\circ}$, especially when the lapse rate becomes smaller, such as in the Arctic. This significant difference in diffusivity angle may result in a highly overestimated window band OLR if the conventional diffusivity angle $\left(52.66^{\circ}\right)$ is used. Although some errors still exist in the spectrally dependent approximation, this approach accounts for the effect of such physical attributes as the total optical depth $\tau_{s}$ and lapse rate and exhibits errors within $5 \%$.

\section{Validation tests and discussion}

In this section, we perform a series of tests with different atmospheric profiles to verify the accuracy of our revised DFA algorithms, in comparison with the conventional constant diffusivity-angle approach. 
In these tests, we calculate the optical depths using a radiative transfer model MODTRAN 5.2, from 100 to $2800 \mathrm{~cm}^{-1}$ at $0.1-\mathrm{cm}^{-1}$ resolution, and then apply a path geometry model that we develop to generate the directional radiance. The path geometry model here can implement either plane-parallel geometry or the realistic path that combines refraction and spherical Earth surface. The realistic path geometry model has been validated against the geometry package of MODTRAN. Note that the realistic path geometry option considers refraction, although its impact is insignificant compared to the spherical geometry, which we focus on here. We angularly integrate the directional radiances from $0^{\circ}$ to $90^{\circ}$ zenith angle at a $0.1^{\circ}$ interval following Eq. (2), at each wavenumber, to generate the reference spectral flux (truth).

In addition to the U.S. standard profile (McClatchey et al. 1972), errors averaged in five bands with different atmospheric profiles are shown in Table 1. Besides the two DFA approaches, we also include a double Gaussian quadrature (2GQ) method ( $\mathrm{Li} \mathrm{2000)}$ in the comparison. The result (Table 1) shows that the 2GQ method generally achieves better accuracy than the constant-angle DFA method (equivalently a $1 \mathrm{GQ}$ method) at the price of doubled computation. However, there is a similar pattern of overestimation in the window band to the constant-angle DFA method when $2 \mathrm{GQ}$ is used, resulting from the neglect of the surface curvature effect. As demonstrated in Figs. $4 \mathrm{c}$ and $4 \mathrm{~d}$, the surface curvature effect may lead to larger than $1 \mathrm{~K}$ of irradiance differences, and it is the major source of error in this case. Failure to account for this effect leads to up to $1.5 \mathrm{~W} \mathrm{~m}^{-2}$ overestimation with the constant-angle DFA approach. To overcome this problem, we propose a similar correction scheme to the Gaussian quadrature (GQ) method in Eq. (A4) in the appendix and generally reduce the error to $0.55 \mathrm{~W} \mathrm{~m}^{-2}$ as presented in Table 2, although $2 \mathrm{GQ}$ shows little improvement compared to the constant-angle DFA method after this correction.

As presented in Fig. 4 and Table 1, the spectrally dependent DFA given in this study approximates the truth very well throughout the spectra. It improves the constantangle DFA approach, and its accuracy is comparable with 2GQ with a plane-parallel geometry in window bands. Compared to the GQ methods, it achieves a noticeable improvement with errors being within $0.50 \mathrm{~W} \mathrm{~m}^{-2}$ in all atmospheric conditions even when a realistic geometry is considered. However, a few caveats should be noted. From the derivation above, it is clear that the solution [Eqs. (29) and (30)] relies on the condition that a constant lapse rate prevails in the vertical range over which the weighting function spans. It means that the DFA approach may incur more uncertainty at the frequencies where the weighting
TABLE 2. As in Table 1, except that a correction to account for Earth's surface geometry is applied.

\begin{tabular}{lccc}
\hline \hline \multicolumn{1}{c}{ Atmosphere type } & Method & {$[760,1000]$} & {$[1061,1230]$} \\
\hline Standard & $\mu_{0}=e^{-1 / 2}$ & -0.35 & -0.13 \\
& $2 \mathrm{GQ}$ & -0.65 & -0.20 \\
Tropical & $\mu_{0}=e^{-1 / 2}$ & -0.40 & -0.11 \\
& $2 \mathrm{GQ}$ & -0.65 & -0.48 \\
Midlatitude summer & $\mu_{0}=e^{-1 / 2}$ & -0.47 & -0.15 \\
& $2 \mathrm{GQ}$ & -0.51 & -0.17 \\
Midlatitude winter & $\mu_{0}=e^{-1 / 2}$ & -0.50 & -0.21 \\
& $2 \mathrm{GQ}$ & -0.63 & -0.18 \\
High-latitude summer & $\mu_{0}=e^{-1 / 2}$ & -0.53 & -0.21 \\
& $2 \mathrm{GQ}$ & -0.78 & -0.25 \\
High-latitude winter & $\mu_{0}=e^{-1 / 2}$ & -0.27 & -0.29 \\
& $2 \mathrm{GQ}$ & -0.61 & -0.17 \\
\hline
\end{tabular}

function peaks near an abrupt change in lapse rates, such as around the tropopause or near a surface temperature inversion layer. The same limitation also exists in the correction of the surface geometry effect in Eq. (28). Moreover, the analytical solutions derived here depend on the $\tau-z$ relation given by Eq. (5). This relation may be obscured by clouds, especially in a partially cloudy sky. In the case of an overcast, thick cloud layer, for example, occurring at the tropopause level, because the relation largely retains above the cloud, we find our approach yields errors within $0.5 \mathrm{~K}$ throughout the middle infrared spectrum.

In conclusion, this study reinvestigates the diffusivity angle approach. We find a physical explanation of why the conventional constant-angle DFA works in the absorption bands and an analytical solution to improve the accuracy in the window bands. This solution takes account of both optical and atmospheric properties while keeping the simplicity of the expression.

Acknowledgments. We thank Jiangnan Li and two anonymous reviewers for their comments. This work is supported by a grant from the Discovery Program of the Natural Sciences and Engineering Research Council of Canada (Grant RGPIN-2019-04511). The source code of this diffusivity angle approach can be downloaded online (https://huanggroup.wordpress.com/ 2018/10/02/diffusivity-angle-script/).

\section{APPENDIX}

\section{Spherical Correction of Gaussian Quadrature Method}

According to Li (2000), quadrature angles are solved from

$$
2 \int_{0}^{1} e^{-\tau / \mu} \mu d \mu=\sum_{i}^{n} b_{i} e^{-\tau / \mu_{0 i}}
$$


A set of weight $b_{i}$ and $\mu_{0 i}$ are given in the referenced literature. When the curved Earth surface is considered, we use $\mu^{\prime}$ as the cosine of the effective zenith angle:

$$
2 \int_{0}^{1} e^{-\tau / \mu^{\prime}} \mu d \mu=\sum_{i}^{n} b_{c i} e^{-\tau / \mu_{0 i}^{\prime}}
$$

where $b_{c i}$ is the corresponding weight. Based on Eq. (28),

$$
\begin{aligned}
& 2 \int_{0}^{1} e^{-\tau / \mu^{\prime}} \mu d \mu=2\left(\frac{R}{R+H}\right)^{2} \int_{0}^{1} e^{-\tau / \mu^{\prime}} \mu^{\prime} d \mu^{\prime}, \\
& 2\left(1-\frac{H}{R+H}\right)^{2} \int_{0}^{1} e^{-\tau / \mu^{\prime}} \mu^{\prime} d \mu^{\prime}=\sum_{i}^{n} b_{c i} e^{-\tau / \mu_{0 i}^{\prime}} .
\end{aligned}
$$

Based on Eq. (27), the cosine of zenith angle at TOA and the adjusted weight $b_{c i}$ are therefore

$$
\begin{aligned}
& \mu_{c i}=\sqrt{1-\left(1-\mu_{0 i}\right)\left(1-\frac{H}{R+H}\right)^{2}}, \\
& b_{c i}=\left(1-\frac{H}{R+H}\right)^{2} b_{i} .
\end{aligned}
$$

\section{REFERENCES}

Armstrong, B., 1968: Theory of the diffusivity factor for atmospheric radiation. J. Quant. Spectrosc. Radiat. Transf., 8, 15771599, https://doi.org/10.1016/0022-4073(68)90052-6.

Bani Shahabadi, M., Y. Huang, L. Garand, S. Heilliette, and P. Yang, 2016: Validation of a weather forecast model at radiance level against satellite observations allowing quantification of temperature, humidity, and cloud-related biases. J. Adv. Model. Earth Syst., 8, 1453-1467, https:// doi.org/10.1002/2016MS000751.
Elsasser, W. M., 1942: Heat Transfer by Infrared Radiation in the Atmosphere. Vol. 6, Harvard Meteorological Studies, Harvard University Press, $107 \mathrm{pp}$.

Feldman, D., W. Collins, and J. Paige, 2015: Pan-spectral observing system simulation experiments of shortwave reflectance and long-wave radiance for climate model evaluation. Geosci. Model Dev., 8, 1943-1954, https://doi.org/10.5194/gmd-8-1943-2015.

Goody, R., and Y. Yung, 1989: Atmospheric Radiation. Oxford University Press, $519 \mathrm{pp}$.

Huang, X., J. N. Cole, F. He, G. L. Potter, L. Oreopoulos, D. Lee, M. Suarez, and N. G. Loeb, 2013: Longwave bandby-band cloud radiative effect and its application in GCM evaluation. J. Climate, 26, 450-467, https://doi.org/10.1175/ JCLI-D-12-00112.1.

Huang, Y., 2013: A simulated climatology of spectrally decomposed atmospheric infrared radiation. J. Climate, 26, 1702-1715, https://doi.org/10.1175/JCLI-D-12-00438.1.

_ , and M. Bani Shahabadi, 2014: Why logarithmic? A note on the dependence of radiative forcing on gas concentration. J. Geophys. Res. Atmos., 119, 13 683-13 689, https://doi.org/ 10.1002/2014JD022466.

— - V. Ramaswamy, X. Huang, Q. Fu, and C. Bardeen, 2007: A strict test in climate modeling with spectrally resolved radiances: GCM simulation versus AIRS observations. Geophys. Res. Lett., 34, L24707, https://doi.org/10.1029/2007GL031409.

Li, J., 2000: Gaussian quadrature and its application to infrared radiation. J. Atmos. Sci., 57, 753-765, https://doi.org/10.1175/ 1520-0469(2000)057<0753:GQAIAT>2.0.CO;2.

McClatchey, R. A., R. W. Fenn, J. A. Selby, F. Volz, and J. Garing, 1972: Optical properties of the atmosphere. Air Force Tech. Rep., 108 pp.

Mehta, A., and J. Susskind, 1999: Outgoing longwave radiation from the TOVS Pathfinder Path A data set. J. Geophys. Res., 104, 12 193-12 212, https://doi.org/10.1029/1999JD900059.

Pan, F., and X. Huang, 2018: The spectral dimension of modeled relative humidity feedbacks in the CMIP5 experiments. J. Climate, 31, 102 021-102 038, https://doi.org/10.1175/JCLID-17-0491.1.

Zhao, J.-Q., and G.-Y. Shi, 2013: An accurate approximation to the diffusivity factor. Infrared Phys. Technol., 56, 21-24, https://doi.org/10.1016/j.infrared.2012.09.003. 